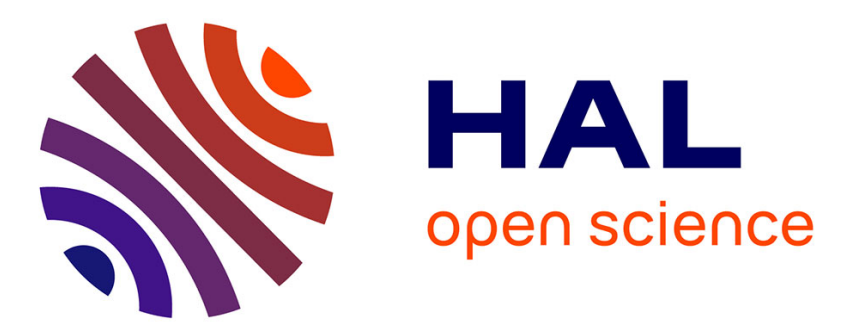

\title{
Enabling the P2P JXTA Platform for High-Performance Networking Grid Infrastructures
}

\author{
Mathieu Jan, Gabriel Antoniu, David A. Noblet
}

\section{To cite this version:}

Mathieu Jan, Gabriel Antoniu, David A. Noblet. Enabling the P2P JXTA Platform for HighPerformance Networking Grid Infrastructures. Intl. Conf. on High Performance Computing and Communications (HPCC'05), Sep 2005, Sorrento, Italy. pp.429-440. inria-00000976

\section{HAL Id: inria-00000976 \\ https://hal.inria.fr/inria-00000976}

Submitted on 9 Jan 2006

HAL is a multi-disciplinary open access archive for the deposit and dissemination of scientific research documents, whether they are published or not. The documents may come from teaching and research institutions in France or abroad, or from public or private research centers.
L'archive ouverte pluridisciplinaire HAL, est destinée au dépôt et à la diffusion de documents scientifiques de niveau recherche, publiés ou non, émanant des établissements d'enseignement et de recherche français ou étrangers, des laboratoires publics ou privés. 


\title{
Enabling the P2P JXTA platform for high-performance networking grid infrastructures
}

\author{
Gabriel Antoniu ${ }^{1}$, Mathieu Jan ${ }^{1}$, and David A. Noblet ${ }^{2}$ \\ 1 IRISA/INRIA \\ Campus de Beaulieu, 35042 Rennes cedex, France \\ Mathieu.Jan@irisa.fr \\ 2 University of New Hampshire, Department of Computer Science \\ Durham, New Hampshire 03824-3591, USA
}

\begin{abstract}
As grid sizes increase, the need for self-organization and dynamic reconfigurations is becoming more and more important, and therefore the convergence of grid computing and Peer-to-Peer (P2P) computing seems natural. Grid infrastructures are generally available as a federation of SAN-based clusters interconnected by high-bandwidth WANs. However, P2P systems are usually running on the Internet, with a non hierarchical network topology, which may raise the issue of the adequacy of the $\mathrm{P} 2 \mathrm{P}$ communication mechanisms on grid infrastructures. This paper evaluates the communication performance of the JXTA P2P platform over high-performance SANs and WANs, for both J2SE and C bindings. We analyze these results and we evaluate solutions able to improve the performance of JXTA on such networking grid infrastructures.
\end{abstract}

Key words: high performance networking, grid computing, P2P, JXTA.

\section{Using P2P techniques to build grids}

Nowadays, scientific applications require more and more resources, such as processors, storage devices, network links, etc. Grid computing provides an answer to this growing demand by aggregating resources made available by various institutions. As their sizes are growing, grids express an increasing need for flexible distributed mechanisms allowing them to be efficiently managed. Such properties are exhibited by Peer-to-Peer (P2P) systems, which have proven their ability to efficiently handle millions of interconnected resources in a decentralized way. Moreover, these systems support a high degree of resource volatility. The idea of using P2P approaches for grid resource management has therefore emerged quite naturally $[1,2]$.

The convergence of $\mathrm{P} 2 \mathrm{P}$ and grid computing can be approached in several ways. For instance, P2P services can be implemented on top of building blocks based on current grid technology (e.g., by using grid services as a communication layer [3]). Conversely, P2P libraries can be used on physical grid infrastructures, for example, as an underlying layer for higher-level grid services [4]. This provides a way to leverage scalable P2P mechanisms for resource discovery, resource replication and fault tolerance. In this paper, we focus on this second approach. 
Using P2P software on physical grid infrastructures is a challenging problem. Grid applications often have important performance constraints that are generally not a usual requirement for $\mathrm{P} 2 \mathrm{P}$ systems. One crucial issue in this context is the efficiency of data transfers. Using P2P libraries as building blocks for grid services, for instance, requires the efficient use of the capacities of the networks available on grid infrastructures: System-Area Networks (SANs) and Wide-Area Networks (WANs). Often, a grid is built as a cluster federation. SANs, such as Giga Ethernet or Myrinet (which typically provide $\mathrm{Gb} / \mathrm{s}$ bandwidth and a few microseconds latency), are used for connecting nodes inside a given high-performance cluster; whereas WANs, with a typical bandwidth of $1 \mathrm{~Gb} / \mathrm{s}$ but higher latency (typically of the order of 10-20 ms), are used between clusters. This is clearly an unusual deployment scenario for P2P systems, which generally target the edges of the Internet (those with low-bandwidth and high-latency links, such as Digital Subscriber Line, or DSL, connections). Therefore, it is important to ask: are $\mathrm{P} 2 \mathrm{P}$ communication mechanisms adequate for a usage in such a context? Is it possible to adapt P2P communication systems in order to benefit from the high potential offered by these high-performance networks?

As an example, JXTA [5] is the open-source project on which, to the best of our knowledge, most of the few attempts for realizing P2P-grid convergence have been based (see the related work below). In its 2.0 version, JXTA consists of a specification of six language- and platform-independent, XML-based protocols that provide basic services common to most $\mathrm{P} 2 \mathrm{P}$ applications, such as peer group organization, resource discovery, and inter-peer communication. To our knowledge, this paper is the first attempt to discuss the appropriateness of using the JXTA P2P platform for high-performance computing on grid infrastructures, by evaluating to what extent its communication layers are able to leverage high-performance (i.e. Gigabit/s) networks. A detailed description of the communications layers of JXTA can be found in [6]. This paper focuses on the evaluation of JXTA-J2SE and JXTA-C ${ }^{3}$ over both SANs and WANs. It also discusses ways to integrate some existing solutions for improving the raw performance.

The remainder of the paper is organized as follows. Section 2 introduces the related work: we discuss some JXTA-based attempts for using P2P mechanisms to build grid services and we mention some performance evaluations of JXTA. Section 3 describes in detail the experimental setup used for both SAN and WAN benchmarks. Sections 4 and 5 present the benchmark results of JXTA over these two types of networks. Finally, Section 6 concludes the paper and discusses some possible future directions.

\section{Related Work}

Several projects have focused on the use of JXTA as a substrate for grid services. The Cog Kit JXTA Project [7] and the JXTA-Grid [8] project are two examples. However, none of these projects are being actively developed and not one has released any prototypes. The Service-oriented Peer-to-Peer Architecture [4] (SP2A) project aims at using $\mathrm{P} 2 \mathrm{P}$ routing algorithms for publishing and discovering grid services. SP2A is based on two specifications: the Open Grid Service Infrastructure (OGSI) and JXTA. None of

\footnotetext{
${ }^{3}$ The only two bindings compliant to JXTA's specifications version 2.0
} 
the projects above has published performance evaluations so far. Finally, JUXMEM [9] proposes to use JXTA in order to build a grid data-sharing service. All of these projects mentioned above share the idea of using JXTA as a low-level interaction substrate over a grid infrastructure. Such an approach brings forth the importance of JXTA's communications performance.

JXTA's communication layers have so far only been evaluated at a cluster level, or over the Internet via DSL connections, but not over grid architectures with high-performance clusters interconnected with high-bandwidth WANs. The performance of JXTA-J2SE communication layers has been the subject of many papers $[10,11,12,13,14,15]$ and has served as reference for comparisons with other P2P systems $[16,17,18]$. The most recent evaluation of JXTA-J2SE is [6], which also provides an evaluation of JXTA-C, but only over Fast Ethernet LAN networks. However, it gives hints on how to use JXTA in order to get good performance on this kind of networks.

\section{Description of the Experimental Setup}

For all reported measurements we use a bidirectional bandwidth benchmark (between two peers), based on five subsequent time measurements of an exchange of 100 consecutive message-acknowledgment pairs sampled at the application level. We chose this test as it is a well-established metric for benchmarking networking protocols, and because of its ability to yield information about important performance characteristics such as bandwidth and latency. Benchmarks were executed using versions 2.2.1 and 2.3.2 of the J2SE binding of JXTA. For the C binding, the CVS head of JXTA-C from the 18th of January 2005 was used. Both bindings were configured to use TCP as the underlying transport protocol. When benchmarks are performed using JXTAJ2SE, the Sun Microsystems Java Virtual Machine (JVM) 1.4.2 is used and executed with - server -Xms 256M - Xmx256M options. The use of other JVMs is explicitly noted and executed with equivalent options. Also note that when the Java binding of JXTA is benchmarked, an additional warm-up phase based on 1000 consecutive message-acknowledgment pairs is performed. Finally, the JXTA-C benchmarks are compiled using gcc 3.3 .3 with the $\mathrm{O} 2$ level of optimization. Tests were performed on the following two types of networks, typically used for building grid infrastructures.

SAN benchmarks. The networks used for the SAN benchmarks are Giga Ethernet and Myrinet (GM driver, version 2.0.11). When the network layer is Myrinet, nodes consist of machines using $2.4 \mathrm{GHz}$ Intel Pentium IV processors, outfitted with $1 \mathrm{~GB}$ of RAM each, and running a 2.4 version Linux kernel. For Giga Ethernet, nodes consist of machines using dual 2.2 GHz AMD Opteron processors, also outfitted with $1 \mathrm{~GB}$ of RAM each, and running a 2.6 version Linux kernel. Since direct communication amongst nodes of a SAN-based cluster is available, direct communications between peers has also been configured. Note that this is allowed by JXTA specifications and therefore does not require the use of additional peers.

WAN benchmarks. The platform used for the WAN benchmarks is the Grid'5000 French national grid platform [19]. Tests were performed between two of the Grid'5000 

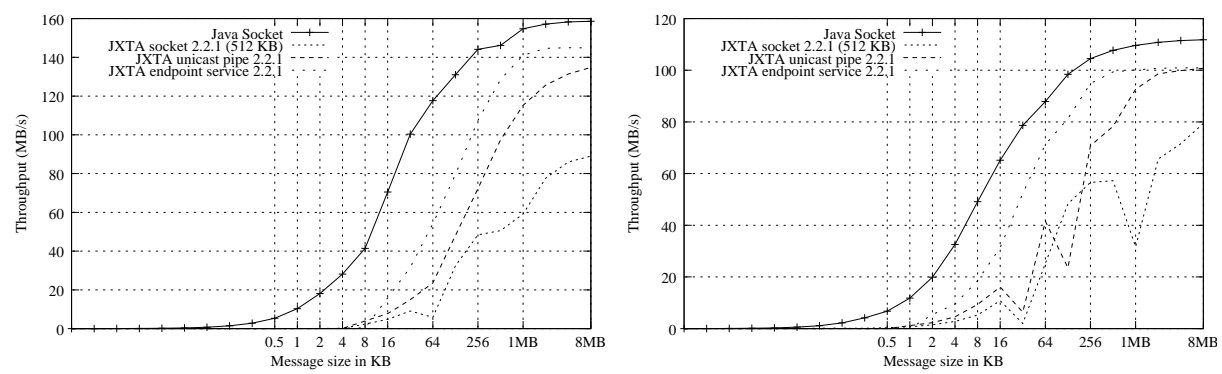

Fig. 1. Bandwidth of each layer of JXTA 2.2.1 as compared to Java sockets over a Myrinet network (left) and a Giga Ethernet network (right).

clusters located in Rennes and Toulouse. On each side, nodes consist of machines using dual 2.2 GHz AMD Opteron processors, outfitted with $1 \mathrm{~GB}$ of RAM each, and running a 2.6 version Linux kernel. The two sites are interconnected through a $1 \mathrm{~Gb} / \mathrm{s}$ link, with an average measured latency of $11.2 \mathrm{~ms}$. Note that as direct communication between nodes is possible within the Grid' 5000 testbed, we configured JXTA peers to enable direct exchanges. As for the SAN benchmarks, this allowed by JXTA specification, even if this is clearly an unusual deployment scenario for P2P systems, where direct communication between peers is the exception rather than the rule (because of firewalls, etc.). However, let us stress that on some grids direct communication is only available between cluster front-ends. In that case, additional evaluations would be necessary.

Communication protocols. JXTA communication layers provide three basic mechanisms for inter-peer communication, with different levels of abstraction. The endpoint service is JXTA's lowest, point-to-point communication layer which provides an abstraction for available underlying transport protocols. Messages sent by this layer are comprised of a series of named and typed message elements [20]. These elements may be required by higher communication layers or added by the application (e.g. the message payload). The pipe service, built on top of the endpoint layer, provides virtual communication channels (or pipes), which are dynamically bound to peer endpoints at runtime, thus allowing developers to abstract themselves from dynamic, runtime changes of physical network addresses. In this paper, we focus on point-to-point pipes, called unicast pipes. Finally, on top of pipes, the JXTA sockets add a data-stream interface, and implement reliability guarantees. JXTA sockets extend the BSD socket API, while still preserving the main feature of pipes: independence from the physical network. However, it should be noted that this layer is not part of the core specifications of JXTA. It is currently only available in JXTA-J2SE.

\section{Performance Evaluation of JXTA over System-Area Networks}

This section analyzes the performance of JXTA's communications layers on SANs. Note that for Myrinet, the Ethernet emulation mode of GM 2.0.11 is used and configured with jumbo frames. This mode allows Myrinet to carry any packet traffic and 

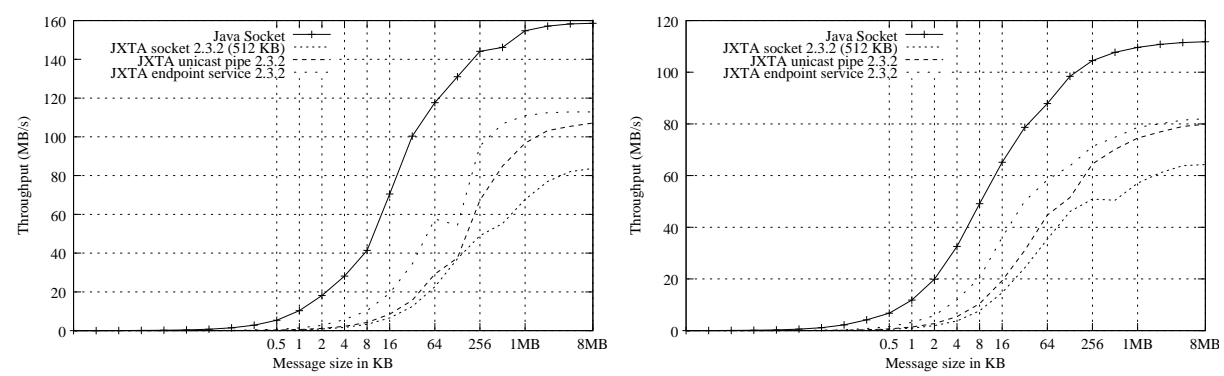

Fig. 2. Bandwidth of each layer of JXTA 2.3.2 as compared to Java sockets over a Myrinet network (left) and a Giga Ethernet network (right).

protocols that can be transported by Ethernet, including TCP/IP. Although this capability is bought at the cost of losing the main advantage of a Myrinet network (e.g. OS-bypass mode), it allows the same socket-based benchmarks to be run unmodified. On this configuration, the bandwidth and latency of plain sockets is around $155 \mathrm{MB} / \mathrm{s}$ and $60 \mu \mathrm{s}$ respectively, whereas on Giga Ethernet it is around $115 \mathrm{MB} / \mathrm{s}$ for the bandwidth and $45 \mu$ s for the latency (average values between $\mathrm{C}$ and Java sockets). These values are used as a reference performance bound.

\subsection{Analysis of JXTA-J2SE's Performance}

\begin{tabular}{|l|c|c|c|c|c|c|}
\hline Version of JXTA & \multicolumn{2}{|c|}{ JXTA-J2SE 2.2.1 } & \multicolumn{2}{|c|}{ JXTA-J2SE 2.3.2 } & \multicolumn{2}{c|}{ JXTA-C } \\
\hline Network & Myrinet & Giga Ethernet & Myrinet & Giga Ethernet & Myrinet & Giga Ethernet \\
\hline Endpoint service & $890 \mu \mathrm{s}$ & $357 \mu \mathrm{s}$ & $624 \mu \mathrm{s}$ & $294 \mu \mathrm{s}$ & $635 \mu \mathrm{s}$ & $322 \mu \mathrm{s}$ \\
\hline Unicast pipe & $1.9 \mathrm{~ms}$ & $834 \mu \mathrm{s}$ & $1.7 \mathrm{~ms}$ & $711 \mu \mathrm{s}$ & $1.7 \mathrm{~ms}$ & $727 \mu \mathrm{s}$ \\
\hline JXTA socket & $3.3 \mathrm{~ms}$ & $1.3 \mathrm{~ms}$ & $2.4 \mathrm{~ms}$ & $977 \mu \mathrm{s}$ & & \\
\hline
\end{tabular}

Table 1. Latency results for JXTA-J2SE and JXTA-C.

JXTA-J2SE endpoint service. Figure 1 shows that the endpoint service of JXTA 2.2.1 nearly reaches the bandwidth of plain sockets over SAN networks: $145 \mathrm{MB} / \mathrm{s}$ over Myrinet and $101 \mathrm{MB} / \mathrm{s}$ over Giga Ethernet. However, Figures 2 also shows that the bandwidth of the JXTA 2.3.2 endpoint layer has decreased: drops of $32 \mathrm{MB} / \mathrm{s}$ over Myrinet and $20 \mathrm{MB} / \mathrm{s}$ over Giga Ethernet are observed. These lower bandwidths affect all versions of JXTA above its release 2.2.1 and are explained by a new implementation of the endpoint layer that shipped with JXTA 2.3. The profiling of JXTA has pointed out that this drop of performance is due to the mechanism used for limiting the size of messages sent by the endpoint layer. Moreover, since JXTA 2.3, the limit has been lowered to $128 \mathrm{~KB}$ of application-level payload (larger messages are dropped). This limitation was introduced into JXTA to in order to promote some fairness in resource sharing among peers on the network, for instance when messages must be stored on relay peers (the type of peer required to exchange messages through firewalls). However, 
as no relay peers are needed when using SANs, we removed this limit. Table 1 shows that latency results of JXTA-J2SE have improved since version 2.2.1. The latency of the JXTA 2.3.2 endpoint service over Giga Ethernet reaches a value under $300 \mu$ s. Moreover, it goes down even further to $268 \mu$ s and $229 \mu$ s when using the SUN 1.5 and IBM 1.4.1 JVMs, respectively. The difference between Myrinet and Giga Ethernet results is due to the hardware employed, as the Ethernet emulation mode is used for Myrinet.

JXTA-J2SE unicast pipe. In addition, Figure 1 and 2 demonstrate a bandwidth degradation for JXTA-J2SE. For example, while JXTA 2.2.1 unicast pipe attains a good peak bandwidth of $136.8 \mathrm{MB} / \mathrm{s}$ over Myrinet, its 2.3.2 counterpart reaches a bandwidth of only $106.5 \mathrm{MB} / \mathrm{s}$. A similar performance degradation can be observed on Giga Ethernet. However, the shape of the curve of unicast pipes 2.2.1 on Giga Ethernet has not been explained so far. We suspect the first drop is due to a scheduling problem. On the other hand, the reason of the drop at $128 \mathrm{~KB}$ of application payload is still unknown. At the same payload size, a smaller drop for JXTA unicast pipes 2.3.2 over Giga Ethernet can be observed, but no link have been established with the previously mentioned drop, as this drop also occurs at the endpoint level. Overall, the small performance degradation as compared to the endpoint layer is explained by the composition of a pipe message: the presence of an XML message element requiring a costly parsing prevents this layer from reaching the performance of the endpoint layer. Moreover, as shown on table 1, this extra parsing required for each pipe message also affects latency results: compared to the endpoint layer, latencies increase by more than $400 \mu \mathrm{s}$. However, unicast pipes are still able to achieve latencies in the sub-millisecond range, at least on Giga Ethernet.

JXTA-J2SE sockets. As opposed to previous layers, JXTA sockets are far from reaching the performance of plain Java sockets. Indeed, in their default configuration (e.g. with an output buffer size of $16 \mathrm{~KB}$ ), JXTA sockets 2.2.1, for instance, attain a peak bandwidth of $12 \mathrm{MB} / \mathrm{s}$ over a Myrinet network. As for unicast pipes, a similar low bandwidth result is reported on Giga Ethernet. We were able to significantly improve the bandwidth and achieve $92 \mathrm{MB} / \mathrm{s}$ by increasing the size of the output buffer to $512 \mathrm{~KB}$, as shown on Figures 1 and 2. As for the unicast pipes, the irregular shape of JXTA sockets 2.2.1 curves has not been explained so far. Again, we suspect the first drop is due to a scheduling problem. The next drop may be due to some message losses when the message size is around the size of the output buffer, since many reliability issues have been fixed up to JXTA 2.3.2. Table 1 highlights the progress being made by JXTA on latency, as only JXTA Sockets 2.3.2 on Giga Ethernet is able to reach a latency under one millisecond.

Discussion. In conclusion, JXTA-J2SE 2.2.1 communication layers are able to nearly saturate SANs, but only at the endpoint and pipe levels. The measurements revealed that the bandwidth of JXTA 2.2.1 is higher than JXTA 2.3.x. Latency results have largely improved since JXTA 2.2.1, but without reaching reasonably good performance for SANs. Finally, this evaluation has also highlighted that, in their default configuration, JXTA sockets achieve a very poor bandwidth. However, this result can significantly be improved by increasing the output buffer size. This requires the JXTA socket programmer to explicitly set this parameter in the user code. Based on these results, we can 
conclude that JXTA-J2SE can be adapted in order to benefit from the potential offered by SANs, at least on the bandwidth side.

\subsection{Analysis of JXTA-C's Performance}
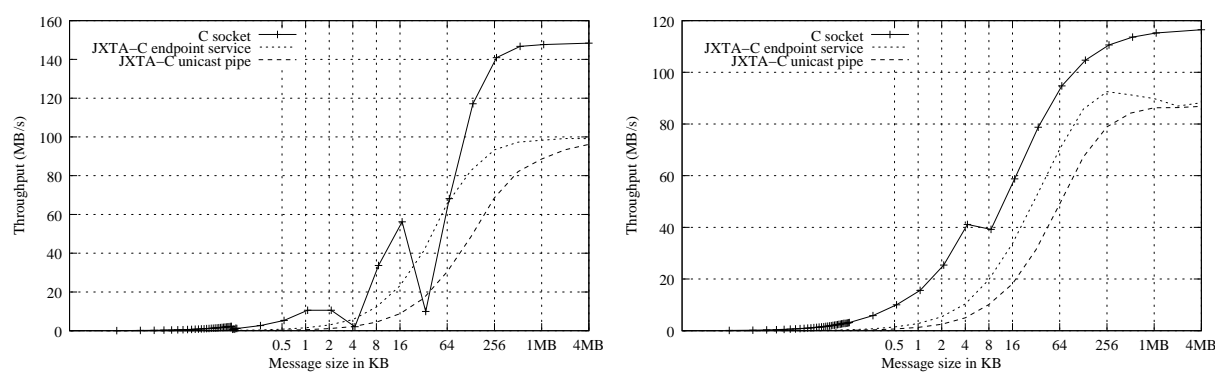

Fig. 3. Bandwidth of each layer of JXTA-C as compared to $\mathrm{C}$ sockets over a Myrinet network (left) and a Giga Ethernet network (right).

Figure 3 shows the bandwidth measurements of all the communications layers of JXTA-C over SANs. Note that, as in the previous section, $\mathrm{C}$ sockets are used as an upper reference bound. The peak bandwidths of the endpoint service over Myrinet and Giga Ethernet are $100 \mathrm{MB} / \mathrm{s}$ and $92.5 \mathrm{MB} / \mathrm{s}$, respectively. The upper layer (unicast pipe) reaches bandwidths of 97.6 MB/s and 87.7 MB/s over Myrinet and Giga Ethernet, respectively. These unsatisfactory results are due to memory copies being used in the implementation of the endpoint layer of JXTA-C. Table 1 highlights reduced latencies, especially on Giga Ethernet, as compared to results published in [6]: $820 \mu$ s for the endpoint layer and $1.99 \mathrm{~ms}$ for the pipe layer. To achieve this improvement, we modified the implementation of the endpoint layer of JXTA-C by disabling TCP packet aggregation mechanism, as it adds significantly to the latency. Consequently, the buffering mechanism is now performed within the endpoint layer allowing one TCP packet to be sent for a single JXTA message with a minimal latency. These modifications have been committed into the CVS of JXTA-C and are publicly available.

Based on this evaluation, we can conclude that, in their current implementation, the communication layers of JXTA-C are not able to saturate SANs. The non-zero copy implementation of the endpoint layer prevents JXTA-C from approaching $\mathrm{Gb} / \mathrm{s}$ bandwidths available over SANs. Note, however, that JXTA-C is in the process of being revived; so we believe that the performance of JXTA-C will increase in the near future such that it will be able to efficiently use SANs.

\subsection{Fully exploiting SAN capacities}

In all previously reported evaluations based on Myrinet, the Ethernet emulation mode of GM is used. However, this removes the ability to by-pass the IP stack of the OS and introduces unneeded overhead. Consequently, communication layers are unable to 
fully exploit the capacities offered by Myrinet: full-duplex bandwidths of nearly $2 \mathrm{~Gb} / \mathrm{s}$ and latencies of less than $7 \mu$ s thanks to zero-copy communication protocols. PadicoTM [21] is a high-performance framework for networking and multi-threading which allows middleware systems to transparently take advantage of such features. In this section, we focus on the virtual sockets feature offered by PadicoTM, which provides a way to directly access GM network interfaces. This is achieved by dynamically mapping, at runtime, standard sockets functions on top of GM API functions, without going through the TCP/IP stack. Zero-copy is therefore possible and allows, for example, plain sockets to transparently reach a bandwidth of more than $230 \mathrm{MB} / \mathrm{s}$ and latency of $15 \mu \mathrm{s}$ on Myrinet, compared to $160 \mathrm{MB} / \mathrm{s}$ and $51 \mu$ s without PadicoTM.

We have successfully ported JXTA-C to PadicoTM, without changing one line of code of JXTA-C. We only performed some minor modifications inside the OSindependent layer used by JXTA-C: the Apache Portable Runtime (APR). We changed from the default Posix thread library on which APR is based, to the Marcel [22] thread library used by PadicoTM. However, these modifications could be automatically achieved by a single sed command. An improvement of $32 \mathrm{MB} / \mathrm{s}$ for the bandwidth of JXTA-C's endpoint layer has been measured resulting in a peak bandwidth of $140 \mathrm{MB} / \mathrm{s}$, thus reaching over $1 \mathrm{~Gb} / \mathrm{s}$. On the latency side, no significant improvements have been observed, as the non-zero copy communication layers prevents JXTA-C from fully benefiting from the OS-bypass feature of Myrinet. Note that we did not use PadicoTM with JXTA-J2SE, since PadicoTM currently supports only the open-source Kaffe JVM. Unfortunately, this JVM is not compliant with Java specification version 1.4 and, therefore, is unable to run the Java binding of JXTA.

In conclusion, our experiments with PadicoTM show that JXTA could fully benefit from the potential performance of SAN networks if: 1) the implementation of all JXTA communication layers should respect a zero-copy policy, 2) PadicoTM adds support for JXTA-compatible JVMs (e.g. compliant to version 1.4 of Java's specifications). However, we believe that these issues will be solved in the near future.

\section{Performance Evaluation of JXTA over Wide-Area Networks}

We performed the same type of measurements on WAN networks. Note that we had to tune network settings of nodes used for this benchmark. Our default maximum TCP buffer size initially set to 131072 bytes was limiting the bandwidth to only $7 \mathrm{MB} / \mathrm{s}$. Based on the bandwidth $*$ delay law, we computed a theoretical maximum size of 1507328 bytes and increased this value by an arbitrary factor of 1.2. Therefore, we set the maximum TCP buffer sizes on each node to 1959526 bytes; ttcp configured with this value measured a raw TCP bandwidth of $107 \mathrm{MB} / \mathrm{s}$, a reasonable level of performance.

JXTA-J2SE's performances. As for SAN Giga Ethernet benchmarks, Figure 4 shows that the endpoint layer and unicast pipes of JXTA-J2SE are able to perform similarly to plain sockets over a high-bandwidth WAN of $1 \mathrm{~Gb} / \mathrm{s}$. This level of performance was reached by modifying JXTA-J2SE's code in order to properly set TCP buffer sizes to 1959526 bytes before binding sockets on both sides. Using the default setting, a bandwidth of only $6 \mathrm{MB} / \mathrm{s}$ was reached for JXTA 2.2 .1 and less than $1 \mathrm{MB} / \mathrm{s}$ for JXTA 2.3.2. 
As opposed to SAN benchmarks, both versions of JXTA-J2SE achieve the same performance. This can be explained by the the fact that the higher latency of WANs hides the cost of the mechanism implemented for limiting the size of JXTA messages. Figures 4 also points out the same performance degradation for JXTA sockets as for SAN benchmarks. However, JXTA socket 2.3.2 achieves a higher bandwidth compared to its 2.2.1 counterpart. Performance drops of unicast pipes and JXTA sockets for JXTA-J2SE 2.2.1 for message size of $4 \mathrm{MB}$ have not been explained so far.
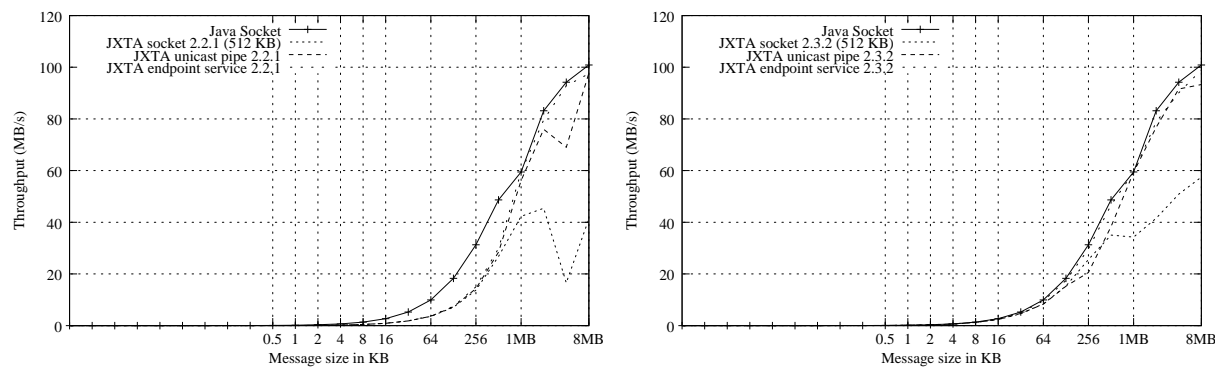

Fig. 4. Bandwidth of each layer for JXTA-J2SE 2.2.1 (left) and 2.3.2 (right) compared to Java sockets over a high-bandwidth WAN.

JXTA-C's performances. Figure 5 shows similar results for the communication layers of JXTA-C over WANs compared to the SAN benchmarks: both layers reach a bandwidth slightly above $80 \mathrm{MB} / \mathrm{s}$, for a message size of $2 \mathrm{MB}$. The observed performance degradation after this message size has not been explained.

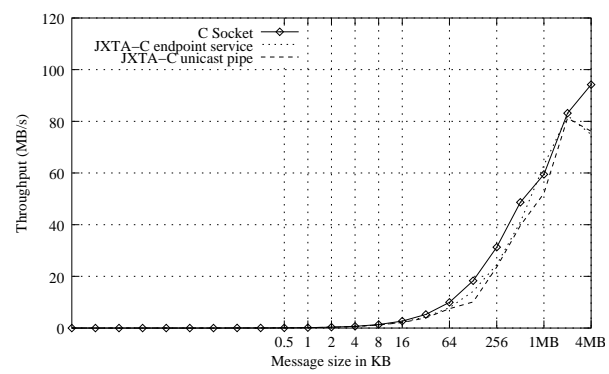

Fig. 5. Bandwidth of each layer for JXTA-C compared to C sockets over a high-bandwidth WAN.

Discussion. Based on this evaluation, we can conclude that JXTA's communication layers, when used on high-bandwidth WANs, are able to reach the same bandwidths as for SAN benchmarks. Both versions of JXTA-J2SE (and not only JXTA-J2SE 2.2.1) are able to efficiently use the bandwidth available on links used for interconnecting sites of a grid, whereas the non-zero copy communication layers prevents JXTA-C from saturating this type of links. 


\section{Conclusion}

In the context of the current efforts for building grid services on top of P2P libraries, an important question is: to what extent is it reasonable to rely on $\mathrm{P} 2 \mathrm{P}$ mechanisms to support the dynamic character of the grid? Are P2P techniques only useful for resource discovery? Or is there a way to take one step further, and efficiently exploit P2P data communication mechanisms? The question of the adequacy of $\mathrm{P} 2 \mathrm{P}$ communication mechanisms for performance-constrained usages is therefore important in this context.

In this paper we show that, if it is correctly tuned, the JXTA platform can deliver adequate performance (e.g., over $1 \mathrm{Gbit} / \mathrm{s}$ bandwidth) and, furthermore, we explain how this performance can be improved thanks to specialized external libraries. First, we evaluated the basic performance of the communication layers of the JXTA generic P2P framework on the Grid5000 testbed. Giga-Ethernet and Myrinet links are used as SANs within each site, while high-bandwidth WANs interconnect the various sites. We then provided an analysis of the performance of the J2SE and C bindings of JXTA over these two types of networks. We show that the JXTA-J2SE 2.2.1 communication layers are able to nearly saturate SANs, at the endpoint and pipe levels, whereas the bandwidth is poor at the JXTA socket level. We also explain how to improve this bandwidth by tuning the TCP output buffer size. Finally, we show that performance can further be improved on SANs by using the PadicoTM environment, which provides a direct access to the API of the Myrinet driver by by-passing the OS and does not require any modifications of JXTA. The overall conclusion of these experiments is that JXTA may provide adequate communication performance that are required by grid computing applications.

However, these evaluations have revealed some weaknesses of JXTA in both the SAN and WAN areas. JXTA-J2SE bandwidths have degraded since JXTA 2.3, hindering JXTA from saturating SAN links. Moreover, the communication layers of JXTA-C do not follow a zero-copy policy, therefore limiting the bandwidth and latency results. Therefore, in spite of our initial efforts, JXTA-C still needs some improvements in order to be able to fully benefit of the available bandwidth provided by SANs. When these issues are solved a bandwidth of over $200 \mathrm{MB} / \mathrm{s}$ should be reached through the use of PadicoTM. On the WAN side, we plan to use parallel streams for both bindings of JXTA, in order to allow an efficient use of high-bandwidth WANs. Again thanks to PadicoTM, this functionality will be transparently available to JXTA-C, whereas for JXTA-J2SE this would require implementing this functionality. Finally, it would also be interesting to measure the impact of on-the-fly compression techniques available in PadicoTM for WAN transfers.

\section{References}

1. Foster, I., Iamnitchi, A.: On Death, Taxes, and the Convergence on Peer-to-Peer and Grid Computing. In: 2nd International Workshop on Peer-to-Peer Systems (IPTPS '03). Number 2735 in Lect. Notes in Comp. Science, Berkeley, CA, Springer-Verlag (2003)

2. Talia, D., Trunfio, P.: Toward a Synergy Between P2P and Grids. IEEE Internet Computing 7 (2003) 94-96

3. Talia, D., Trunfio, P.: A P2P Grid Services-Based Protocol: Design and Evaluation. In: Euro-Par 2004: Parallel Processing. Number 3149 in Lect. Notes in Comp. Science, Pisa, Italy, Springer-Verlag (2004) 1022-1031 
4. Amoretti, M., Conte, G., Reggiani, M., Zanichelli, F.: Service Discovery in a Grid-based Peer-to-Peer Architecture. In: International Workshop on e-Business and Model Based IT Systems Design, Saint Petersburg, Russia (2004)

5. The JXTA project. http://www - jxta.org/

6. Antoniu, G., Hatcher, P., Jan, M., Noblet, D.A.: Performance Evaluation of JXTA Communication Layers. In: 5th International Workshop on Global and Peer-to-Peer Computing (GP2PC '05), Cardiff, UK (2005) Held in conjunction with the 5th IEEE/ACM International Symposium on Cluster Computing and the Grid (CCGrid '2005).

7. Cog Kit JXTA project. http://www-unix.globus.org/cog/projects/jxta/

8. JXTA-Grid project. http://jxta-grid.jxta.org/

9. Antoniu, G., Bougé, L., Jan, M.: JuxMem: Weaving together the P2P and DSM paradigms to enable a Grid Data-sharing Service. Kluwer Journal of Supercomputing (2005) To appear.

10. Halepovic, E., Deters, R.: The Cost of Using JXTA. In: 3rd International Conference on Peerto-Peer Computing (P2P '03), Linköping, Sweden, IEEE Computer Society (2003) 160-167

11. Halepovic, E., Deters, R.: JXTA Performance Study. In: IEEE Pacific Rim Conference on Communications, Computers and Signal Processing (PACRIM '03), Victoria, B.C., Canada, IEEE Computer Society (2003) 149-154

12. Seigneur, J.M.: Jxta Pipes Performance. (2002)

13. Seigneur, J.M., Biegel, G., Jensen, C.D.: P2P with JXTA-Java pipes. In: 2nd international Conference on Principles and Practice of Programming in Java (PPPJ '03), Kilkenny City, Ireland, Computer Science Press, Inc. (2003) 207-212

14. Halepovic, E., Deters, R.: JXTA Messaging: Analysis of Feature-Performance Tradeoffs. Submitted for publication (2005)

15. Shudo, K., Tanaka, Y., Sekiguchi, S.: P3: Personal Power Plant. GGF10: Open Grid Service Architecture - Peer-to-Peer Research Group (OGSA-P2P RG) (2004)

16. Baehni, S., Eugster, P.T., Guerraoui, R.: OS Support for P2P Programming: a Case for TPS. In: 22nd International Conference on Distributed Computing Systems (ICDCS '02), Vienna, Austria, IEEE Computer Society (2002) 355-362

17. Junginger, M., Lee, Y.: The Multi-Ring Topology - High-Performance Group Communication in Peer-to-Peer Networks. In: 2nd International Conference on Peer-to-Peer Computing (P2P '02), Linköping, Sweden, IEEE Computer Society (2002) 49-56

18. Tran, P., Gosper, J., Yu, A.: JXTA and TIBCO Rendezvous - An Architectural and Performance Comparison. (2003)

19. Grid' 5000 project. http://www.grid5000.org/

20. JXTA specification project. http://spec. jxta.org/

21. Denis, A., Pérez, C., Priol, T.: PadicoTM: An Open Integration Framework for Communication Middleware and Runtimes. Future Generation Computer Systems 19 (2003) 575-585

22. Danjean, V., Namyst, R., Russell, R.: Integrating Kernel Activations in a Multithreaded Runtime System on Linux. In: Parallel and Distributed Processing. Proc. 4th Workshop on Runtime Systems for Parallel Programming (RTSPP '00). Volume 1800 of Lect. Notes in Comp. Science., Cancun, Mexico, In conjunction with IPDPS 2000. IEEE TCPP and ACM, Springer-Verlag (2000) 1160-1167 